\title{
Thermoelectric properties of junctions between metal and strongly correlated semiconductor
}

\author{
Massimo Rontani \\ INFM and Dipartimento di Fisica, Università degli Studi di Modena e Reggio Emilia, 41100 Italy, \\ and Department of Physics, University of California San Diego, La Jolla, California 92093-0319 \\ L. J. Sham \\ Department of Physics, University of California San Diego, La Jolla, California 92093-0319
}

(13 June 2000)

\begin{abstract}
We propose a junction of metal and rare-earth compound semiconductor as the basis for a possible efficient low-temperature thermoelectric device. If an overlayer of rare earth atoms differing from the bulk is placed at the interface, very high values of the figure of merit $Z T$ can be reached at low temperature. This is due to sharp variation of the transmission coefficient of carriers across the junction at a narrow energy range, which is intrinsically linked to the localized character of the overlayer $f$-orbital.
\end{abstract}

New thermoelectric coolers and power generators are attracting increasing interest 1 Their quality is governed by the dimensionless figure of merit $Z T$, a product of transport coefficients to be defined below times the absolute temperature. At present, the best $Z T$ obtainable is $\sim 1$ at room temperature, while a value about $3 \sim 4$ is considered a real breakthrough.

There are two theoretical ideas for increasing $Z T$. One is to utilize the sharp energy features in bulk materials such as strongly correlated semiconductors. other is to exploit the interface energy structures $A$ in metal/superconductor junctions, which have been tested experimentally.

In this Letter, we combine these two ideas in exploring the thermopower behavior of a junction between a metal and a particular class of mixed-valent semiconductors with high dielectric constants, such as $\mathrm{SmB}_{6}$ and $\mathrm{Sm}_{2} \mathrm{Se}_{3}$, called the electronic ferroelectrics (FE) 6 Various cases of the junction are considered, including clean and "dirty" interfaces. When a suitable rare-earth impurity layer forms the interface, we find very high values of $Z T$ at low temperatures. There is great interest in low temperature thermoelectrics for special applications.

Mixed-valent semiconductors are rare-earth compounds, usually cubic, whose relevant electronic properties may be modeled by a $f$-flat band and a broad conduction band, with two electrons per unit cell. Strong correlation between $d$-electrons and $f$-holes can renormalize the bands and create a temperature dependent small gap [see Fig. [1](b)]: we consider this case for the semiconductor on one side of the junction, and describe it with the self-consistent mean field solution (MF) of the FalicovKimball model. 8 The ground state of the insulating phase is found to be a coherent condensate of $d$-electron and $f$ hole pairs, giving a net built-in macroscopic polarization which breaks the crystal inversion symmetry and makes the material ferroelectric.6

While previous calculations have dealt with metal/ordinary-semiconductor junctions neglecting the dependence of the transmission coefficient on the carrier energy, $\mathrm{l}$, 0 for the metal/FE junction this feature turns out to be crucial, requiring a careful treatment.
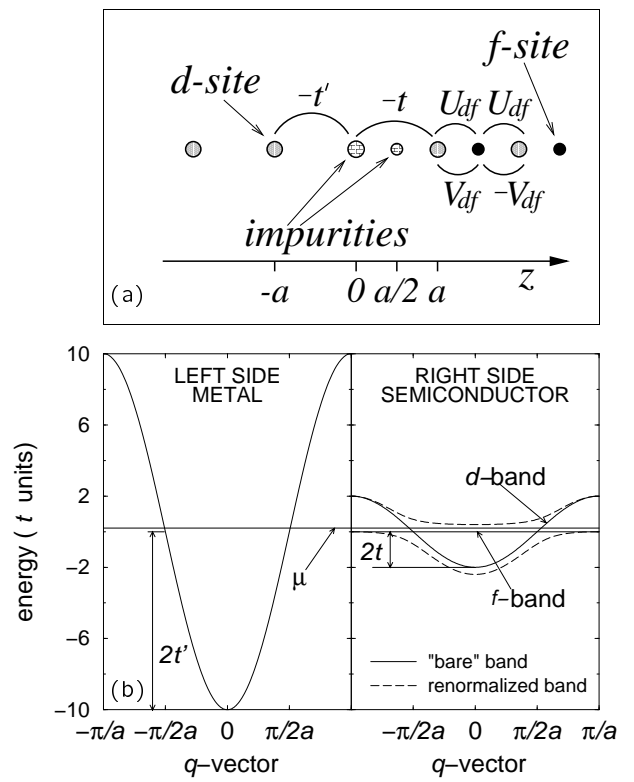

FIG. 1. (a) Pictorial representation of the junction along the $z$ axis perpendicular to the interface. (b) "Bare" band structure $(\Delta=0)$ of materials on both sides of the junction (solid lines), and renormalized bands (dashed lines) at $T=0$. Parameters are $t^{\prime}=5 t$ and $\Delta(T=0)=0.49 t$.

To this aim, we model the motion along the $z$ direction perpendicular to the interface with the one dimensional spinless hamiltonian $\mathcal{H}$, given by the sum of three terms describing the left bulk metal, the interface layer, and the right bulk FE, respectively [see Fig. [1(a)]:

$$
\mathcal{H}=\mathcal{H}_{\text {metal }}+\mathcal{H}_{\text {interface }}+\mathcal{H}_{\mathrm{FE}}
$$


$\mathcal{H}_{\text {metal }}$ is a tight-binding hamiltonian for lattice sites at $z=a j, j<0$ ( $a=$ lattice constant), with energy $\varepsilon_{d}^{\prime}$ and hopping parameter $t$. $\mathcal{H}_{\mathrm{FE}}$ is the bulk Falicov-Kimball hamiltonian:

$$
\begin{aligned}
& \mathcal{H}_{\mathrm{FE}}=\tilde{\varepsilon}_{d} \sum_{j>0} d_{j}^{\dagger} d_{j}+\tilde{\varepsilon}_{f} \sum_{j>0} f_{j+1 / 2}^{\dagger} f_{j+1 / 2}-t \sum_{j \geq 0} d_{j}^{\dagger} d_{j+1} \\
& -V_{d f} \sum_{j \geq 0} f_{j+1 / 2}^{\dagger} d_{j}+V_{d f} \sum_{j>0} f_{j-1 / 2}^{\dagger} d_{j}+\text { H.c. } \\
& +U_{d f} \sum_{j \geq 0} d_{j}^{\dagger} d_{j} f_{j+1 / 2}^{\dagger} f_{j+1 / 2}+U_{d f} \sum_{j>0} d_{j}^{\dagger} d_{j} f_{j-1 / 2}^{\dagger} f_{j-1 / 2} .
\end{aligned}
$$

Here, while electrons can tunnel between $d$-sites with hopping coefficient $t \ll t^{\prime}, f$-electrons are completely localized $\left[f_{j+1 / 2}\left(d_{j}\right)\right.$ destroys a $f$ - $(d$-)electron at $z=$ $(j+1 / 2) a(j a)$ with energy $\left.\tilde{\varepsilon}_{f}\left(\tilde{\varepsilon}_{d}\right)\right]$, but interact with $d$-electrons via the Coulomb repulsion energy $U_{d f}$. Moreover, the $d$ - $f$ hybridization $V_{d f}$ is odd under inversion. On this side of the junction we assume one electron per unit cell. The hamiltonian term

$$
\mathcal{H}_{\text {interface }}=\tilde{\varepsilon}_{d 0} d_{0}^{\dagger} d_{0}+\tilde{\varepsilon}_{f 1 / 2} f_{1 / 2}^{\dagger} f_{1 / 2}
$$

accounts for the possibility of impurity atomic layers at the interface, namely one at $z=0$ made of $d$-like sites with energy $\tilde{\varepsilon}_{d 0}$ and another one at $z=a / 2$ made of $f$-sites with localized orbitals of energy $\tilde{\varepsilon}_{f 1 / 2}$. In our model, the electrostatic potential $V$ across the junction (by applying an external bias) is constant in the two bulk regions (the small gap FE semiconductor has carriers at finite temperature) and has a sharp step at the interface. We adopt the following strategy to solve the motion across the junction: (i) We apply the MF approximation to the hamiltonian $\mathcal{H} \rightarrow \mathcal{H}^{\mathrm{MF}}$, introducing the built-in coherence of the FE bulk $\Delta=U_{d f}<d_{j}^{\dagger} f_{j \pm 1 / 2}>$ (the vacuum is the state with no $f$-holes), 6 treating renormalized site energies $\left(\tilde{\varepsilon}_{d} \rightarrow \varepsilon_{d}, \tilde{\varepsilon}_{f} \rightarrow \varepsilon_{f}\right)$ as material parameters, and choosing $\varepsilon_{d}^{\prime}=\varepsilon_{d}=\varepsilon_{f}=0$, namely the middle of the $d$-band on both sides of the junction and the flat $f$ band are aligned [see Fig. 1(b)]. (ii) We calculate the site coefficients for the Bogoliubov-Valatin operator 11

$$
\gamma_{k e}^{\dagger}=\frac{1}{\sqrt{N_{s}}} \sum_{j}\left\{u_{k}(j) d_{j}^{\dagger}+v_{k}(j+1 / 2) f_{j+1 / 2}^{\dagger}\right\}
$$

$\left(N_{s}=\right.$ number of $d$-sites $)$ which creates elementary electronic excitations of energy $\omega(k)>0$ (referred to the chemical potential $\mu$ ) if applied to the ground state, i.e.

$$
\mathrm{i} \hbar \dot{\gamma}_{k e}=\left[\gamma_{k e}, \mathcal{H}^{\mathrm{MF}}-\mu N\right]=\omega(k) \gamma_{k e},
$$

where $N$ is the number operator, and $k$ is a suitable quantum number 12 Similar expressions occur for holes. From Eqs. (4) and (5) one derives equations for the amplitudes of the quasi-particle excitation $\left(u_{k}(j), v_{k}(j+1 / 2)\right)$.

Solutions are travelling waves partly reflected and partly transmitted at the interface. In particular, the incoming and reflected wave $\left(\Psi_{1 L}, \Psi_{2 L}\right)$ is a compatible solution for $j<0$ plus $u_{k}(0)=\Psi_{1 L}(0)$ if $\Psi_{1 L}(j)=$ $\exp (\mathrm{i} q a j)-R_{k} \exp (-\mathrm{i} q a j), \Psi_{2 L}(j+1 / 2)=0, \omega(q)=$ $e V-2 t^{\prime} \cos (q a)-\mu$, namely the tight-binding solution of the left side metal. Similarly, the transmitted wave

$$
\left(\begin{array}{c}
\Psi_{1 R}(j) \\
\Psi_{2 R}(j+1 / 2)
\end{array}\right)=T_{k}\left(\begin{array}{c}
u_{k} \\
v_{k} \mathrm{e}^{\mathrm{i} k a / 2}
\end{array}\right) \mathrm{e}^{\mathrm{i} k a j}
$$

is a solution of the bulk FE for $j>0$ only if $\omega(k)=$ $\xi_{k}+E_{k}-\mu$, with $\xi_{k}=\varepsilon_{k} / 2, \varepsilon_{k}=-2 t \cos (k a), E_{k}=$ $\left[\xi_{k}^{2}+\left|\Delta_{k}\right|^{2}+\left|V_{k}\right|^{2}\right]^{1 / 2}$, where $\Delta_{k}=2 \Delta \cos (k a / 2)$ and $V_{k}=2 \mathrm{i} V_{d f} \sin (k a / 2)$ have even and odd parity, respectively, and $2\left|u_{k}\right|^{2}=1+\xi_{k} / E_{k},\left|u_{k}\right|^{2}+\left|v_{k}\right|^{2}=1$. Figure 1 (b) shows the quasi-particle band structure on both sides of the junction when $V_{d f} \rightarrow 0$. Note that the FE gap is indirect and that the bottom of conduction band is much flatter than the top of valence band.

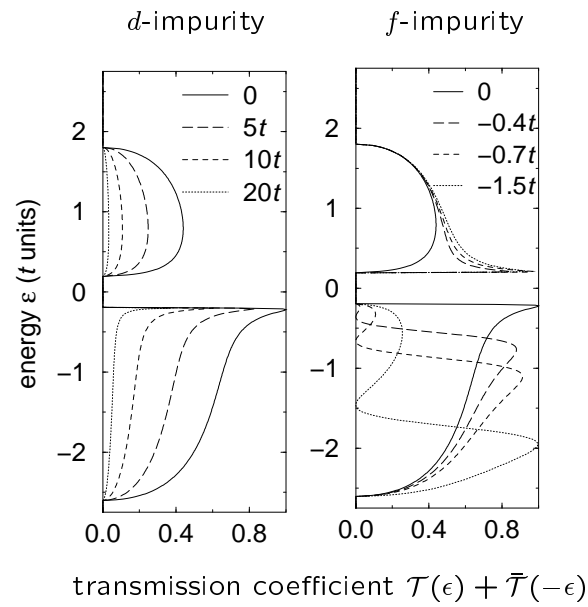

FIG. 2. Total (electron plus hole) transmission coefficient $\mathcal{T}(\epsilon)+\overline{\mathcal{T}}(-\epsilon)$ vs energy, for different values of impurity levels $\varepsilon_{d 0}\left(\varepsilon_{f 1 / 2}\right)$. Left panel: $d$-type impurity. Right panel: $f$-like impurity.

We now have to match left and right bulk solutions at the interface. Because quantities $v_{k}(1 / 2), T_{k}$, and $R_{k}$ are still unknown, the finite-difference equations of motion coming from Eqs. (4) and (5) not yet employed are sqlved, with the elastic scattering condition $\omega(q)=\omega(k) .13$ The reflection coefficient $\mathcal{R}(\omega)$, defined as the ratio of reflected to incident flux, is simply $\left|R_{k}\right|^{2}(q, k>0)$, and the transmission coefficient is $\mathcal{T}(\omega)=1-\mathcal{R}(\omega)$.

Electric and heat density currents are calculated to first order in the differences of $V$ and $T$ on the two sides of the junction. From these the conductance $G=2 e^{2} L_{0} / h$, the thermal conductance $G_{T}=2\left(L_{2}-L_{1}^{2} / L_{0}\right) /(T h)$, and the themopower $Q=L_{1} /\left(e T L_{0}\right)$ of the interface are derived 14 where

$$
L_{n}=\int_{-\infty}^{\infty} \mathrm{d} \epsilon\{\mathcal{T}(\epsilon)+\overline{\mathcal{T}}(-\epsilon)\} \epsilon^{n}\left[-\frac{\partial f(\epsilon)}{\partial \epsilon}\right]
$$


$f(x)$ is the Fermi distribution function, and $\mathcal{T}(x)[\overline{\mathcal{T}}(x)]$ is the electron (hole) transmission coefficient, non-zero only for positive excitation energies within the band range. The interface figure of merit is $Z T=Q^{2} G T / G_{T}$.

Figure 2 shows the total transmission coefficient $\mathcal{T}(\epsilon)+$ $\overline{\mathcal{T}}(-\epsilon)$ vs energy for different values of impurity levels $\varepsilon_{d 0}$ (left panel) and $\varepsilon_{f 1 / 2}$ (right panel). We consider a system at $T=0$ with the same parameters of Fig. 1(b), i.e. one in which the metal band is much broader than the semiconductor one and the FE band gap is $0.4 t$, approximately one tenth of the total bandwidth $\left(V_{d f} \rightarrow 0\right)$. One sees that the clean interface $\left(\varepsilon_{d 0}=\varepsilon_{f 1 / 2}=0\right)$ already presents a strong electron-hole asymmetry. Hence, from Eq. (7) high values of $Q$ follow. The effect of an impurity $d$-layer is just to uniformly depress $\mathcal{T}+\overline{\mathcal{T}}$ : the greater the impurity level energy, the lower the transmission (left panel). This trend, whose global effect is found to enhance $Z T$, has intrinsic physical limitations because $\varepsilon_{d 0}$ must be of the same order of magnitude as bulk energies in realistic systems. The behavior of an $f$-impurity is drastically different. We see that (right panel), as we set $\varepsilon_{f 1 / 2}$ to negative values, $\overline{\mathcal{T}}$ goes to zero in the neighborhood of the same values, as if the hole were completely backscattered from the interface when resonating with the impurity atom. A similar behavior occurs for $\varepsilon_{f 1 / 2}>0$. The overall effect is so strong even to change the dominant (electron or hole) character of transport and, hence, the sign of $Q$.

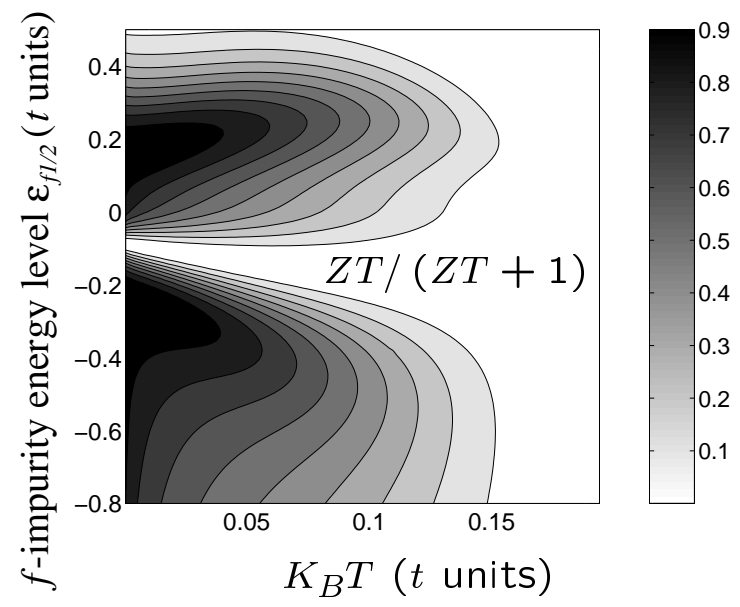

FIG. 3. Two-dimensional contour plot of $Z T /(Z T+1)$ in the $T-\varepsilon_{f 1 / 2}$ plane. The gray scale goes from 0 (white) to 1 (black), and the contour lines are incremented by 0.1 . $\mathrm{Pa}$ rameters as in Fig. 1 .

The effect of the $f$-impurity layer on $Z T$ is shown in Fig. 3. We represent the quantity $Z T /(Z T+1)$ (due to the huge range of $Z T)$ as a contour plot in the $T-\varepsilon_{f 1 / 2}$ plane. While $Z T$ has no upper bound, $Z T /(Z T+1) \leq 1$. For a fixed value of $\varepsilon_{f 1 / 2}, Z T$ goes to zero as $T$ approaches the critical temperature $T_{c}\left(K_{B} T_{c}=0.19 t\right.$ here $)$ at which the gap vanishes and the bulk FE turns into a metal $[\mathcal{T}(\epsilon)=\overline{\mathcal{T}}(-\epsilon)]$. In the opposite limit $T \rightarrow 0, Z T$ can reach very high values (the darkest region enclosed by the inner contour line corresponds to $Z T>90$ ), depending on $\varepsilon_{f 1 / 2}$. At $T>T_{c} Z T$ goes to zero, as $\varepsilon_{f 1 / 2}$ varies, when $Q$ changes sign.

From Fig. 3, there is an optimal value of $\varepsilon_{f 1 / 2}$ of the interafce layer at each temperature. The corresponding maxmimum figure of merit $Z T$ increases with lower temperature. For example, with a gap of $100 \mathrm{meV}$ at $T=0$, the best value of $Z T$ will be $\sim 1$ at $T=300 \mathrm{~K}$, but already $\sim 6$ at $150 \mathrm{~K}$ and $\sim 100$ at $40 \mathrm{~K}$.

In summary, we have made a qualitative theoretical study of the possibility that a junction of metal and FE (as opposed to bulk materials) produces high thermopower. The figure of merit $Z T$ attained is very high, especially at low $T$. In these regimes, bulk thermal conductivity would be dominated by phonons which would reduce $Z T$. However, if we choose two materials with large thermal impedance mismatch, the phonon scatterings at the interface would decrease the junction thermal conductivity. 15 Thus, phonon conductivity would not vitiate the high $Z T$ found. In a longer paper, we will consider the role of phonons in greater detail and include a more comprehensive study of junctions with different classes of semiconducting materials.

Work supported by the NSF contract DMR 9721444, INFM PRA99-SSQI, and Progetto Giovani Ricercatori. We thank D. Chemla, M. L. Cohen and S. G. Louie for the hospitality at University of California, Berkeley, where this work was carried out. L. J. S. thanks J. E. Hirsch for helpful discussions.

${ }^{1}$ G. Mahan, B. Sales and J. Sharp, Phys. Today 50 (3), 42 (1997); G. D. Mahan, Solid State Physics 51, (1998), p 81.

${ }^{2}$ G. D. Mahan and J. O. Sofo, Proc. Natl. Acad. Sci. USA, 93, 7436 (1996).

${ }^{3}$ W. Mao and K. S. Bedell, Phys. Rev. B 59, R15590 (1999).

${ }^{4}$ H. L. Edwards, Q. Niu, G. A. Georgakis, and A. L. de Lozanne, Phys. Rev. B 52, 5714 (1995).

${ }^{5}$ M. Nahum, T. M. Eiles, and J. M. Martinis, Appl. Phys. Lett. 65, 3123 (1994).

${ }^{6}$ T. Portengen, Th. Östreich, and L. J. Sham, Phys. Rev. Lett. 76, 3384 (1996); Phys. Rev. B 54, 17452 (1996).

${ }^{7}$ G. Aeppli and Z. Fisk, Comments Cond. Mat. Phys. 16, 155 (1992).

${ }^{8}$ L. M. Falicov and J. C. Kimball, Phys. Rev. Lett. 22, 997 (1969).

${ }^{9}$ G. D. Mahan and L. M. Woods, Phys. Rev. Lett. 80, 4016 (1998).

10 B. Moyzhes and V. Nemchinsky, Appl. Phys. Lett. 73, 1895 (1998).

${ }^{11}$ P. G. de Gennes, Superconductivity in Metals and Alloys, 
W. A. Benjamin, New York (1966), chap. 5.

${ }^{12}$ In our calculation $\Delta(T)$ and $\mu(T)$ are bulk FE values determined self-consistently.

13 J. E. Hirsch, Phys. Rev. B 50, 3165 (1994).

14 J. M. Ziman, Electrons and Phonons, Oxford, London (1960), chap. VII.

${ }^{15}$ S. M. Lee, D. G. Cahill, and R. Ventakasubramanian, Appl. Phys. Lett. 70, 2957 (1997). 